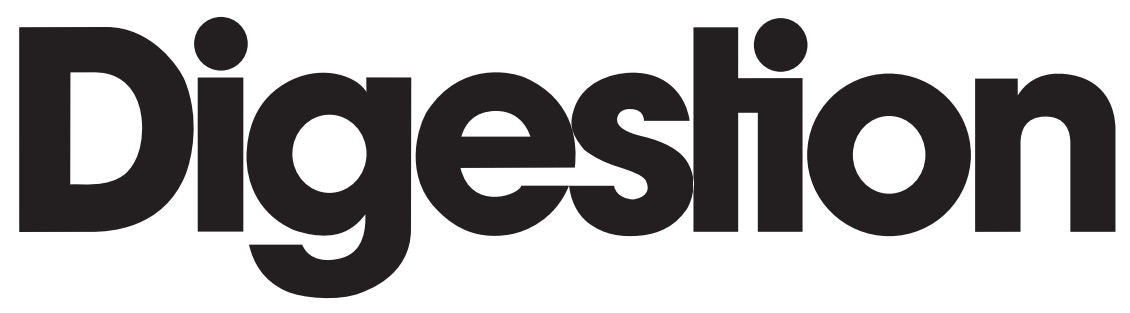

\title{
International Journal of Gastroenterology
}

Founded as 'Archiv für Verdauungskrankheiten' 1895 by I. Boas

Continued as 'Gastroenterologia' 1939-1967

Former Editors: P. Morawitz (1934-1936), R. Staehelin (1937-1943), A. Hurst (1940-1945),

W. Löffler (1943-1961), T.C. Hunt (1947-1967), N. Henning (1953-1962), B. Ihre (1953-1967),

H. Bartelheimer (1963-1967), M. Demole (1963-1971), H. Kapp (1968-1970),

R. Lambert (1972-1978), W. Creutzfeldt (1979-1992), R. Arnold (1993-2003)

\section{Editors-in-Chief}

C. Beglinger, Basel (Switzerland)

B. Göke, Munich (Germany)
Official Journal of

The Japanese

Gastroenterological Association (JGA)

\section{JGA Editorial Representatives}

T. Yoshikawa, Kyoto

K. Takeuchi, Kyoto

\section{Associate Editors}

N. Arber, Tel Aviv (Israel)

G.A. Kullak-Ublick, Zurich (Switzerland)

P. Michetti, Lausanne (Switzerland)

C. Sakamoto, Tokyo (Japan)

R.M. Schmid, Munich (Germany)

J. Tack, Leuven (Belgium) 
S. Karger

Medical and Scientific Publishers

Basel $\cdot$ Freiburg $\cdot$ Paris $\cdot$ London .

New York $•$ Bangalore $\cdot$ Bangkok .

Shanghai $\cdot$ Singapore $\cdot$ Tokyo $\cdot$ Sydney
Disclaimer

The statements, options and data contained in this publication are solely those of the individual authors and contributors and not of the publisher and the editor(s). The appearance of advertisements in the journal is not a warranty, endorsement or approval of the products or services advertised or of thei effectiveness, quality or safety. The publisher and the editor(s) disclaim responsibility for any injury to persons or property resulting from any ideas, methods, instructions or products referred to in the content or advertisements.

Drug Dosage

The authors and the publisher have exerted every effort to ensure that drug selection and dosage set forth in this text are in accord with current recommendations and practice at the time of publication. However, in view of ongoing research, changes in government regulations, and the constant flow of information relating to drug therapy and drug reactions, the reader is urged to check the package insert for each drug for any change in indications and dosage and for added warnings and precautions. This is particularly important when the recommended agent is a new and/or infrequently employed drug.
All rights reserved.

No part of this publication may be translated into other languages, reproduced or utilized in any form or by any means, electronic or mechanical, including photocopying, recording, microcopying, or by any information storage and retrieval system, without permission in writing from the publisher or, in the case of photocopying, direct payment of a specified fee to the Copyright Clearance Center (see 'General Information').

(c) Copyright 2008 by S. Karger AG

P.O. Box, CH-4009 Basel (Switzerland)

Printed in Switzerland

on acid-free and non-aging paper (ISO 9706) by

Reinhardt Druck, Basel

\section{KARGER}

Fax +4161306 1234

E-Mail karger@karger.ch

www.karger.com 


\section{Digestion}

\section{No. 1}

Colorectal Cancer Can Be Prevented

Guest Editor: Nadir Arber, Tel Aviv

Editorial

5 Colorectal Cancer Can Be Prevented Arber, N. (Tel Aviv)

7 Can We Identify the High-Risk Patients to Be Screened? A Genetic Approach Gammon, A.; Kohlmann, W.; Burt, R. (Salt Lake City, Utah)

20 Colorectal Cancer Screening by Colonoscopy - Current Issues Kaminski, M.F.; Regula, J. (Warsaw)

26 New Stool Screening Tests for Colorectal Cancer Young, G.P.; Cole, S. (Adelaide)

34 CT Colonography (Virtual Colonoscopy): Technique, Indications and Performance Blachar, A. (Tel Aviv/Pittsburgh, Pa.); Sosna, J. (Jerusalem/Boston, Mass.)

42 The New Scopes - Broadening the Colonoscopy Marketplace Rösch, T. (Berlin); Eickhoff, A. (Ludwigshafen); Fritscher-Ravens, A. (London); Eliakim, R. (Haifa); Arber, N. (Tel-Aviv)

51 Chemoprevention of Colorectal Cancer Das, D. (Leicester); Arber, N. (Tel Aviv); Jankowski, J.A. (Leicester/Oxford)

68 Prevention of Colorectal Cancer in High-Risk Populations: The Increasing Role for Endoscopy and Chemoprevention in FAP and HNPCC

Lynch, P.M. (Houston, Tex.)

77 Author and Subject Index
No. 2

Therapy of Crohn's Disease - Update 2008

Guest Editor: Florian Froehlich, Lausanne

83 Preface

84 EPACT II: Project and Methods

Juillerat, P. (Lausanne); Froehlich, F. (Lausanne/Basle); Felley, C.; Pittet, V.; Mottet, C.; Gonvers, J.-J.; Michetti, P.; Vader, J.-P. (Lausanne)

92 Mild-to-Moderate Active Luminal Crohn's Disease Michetti, P.; Juillerat, P.; Mottet, C.; Pittet, V.; Gonvers, J.-J.; Vader, J.-P. (Lausanne); Froehlich, F. (Lausanne/Basle); Felley, C. (Lausanne)

99 Severe and Steroid-Resistant Crohn's Disease Michetti, P.; Mottet, C.; Juillerat, P.; Pittet, V.; Felley, C.; Vader, J.-P.; Gonvers, J.-J. (Lausanne); Froehlich, F. (Lausanne/Basle)

109 Fistulizing Crohn's Disease Felley, C.; Mottet, C.; Juillerat, P.; Pittet, V. (Lausanne); Froehlich, F. (Lausanne/Basle); Vader, J.-P.; Michetti, P.; Gonvers, J.-J. (Lausanne)

113 Fibrostenotic Crohn's Disease Froehlich, F. (Lausanne/Basle); Juillerat, P.; Mottet, C.; Pittet, V.; Felley, C.; Vader, J.-P.; Gonvers, J.-J.; Michetti, P. (Lausanne)

116 Maintenance of Medically Induced Remission of Crohn's Disease

Gonvers, J.-J.; Juillerat, P.; Mottet, C.; Pittet, V.; Felley, C.; Vader, J.-P.; Michetti, P. (Lausanne); Froehlich, F. (Lausanne/Basle)

130 Maintenance of Surgically Induced Remission of Crohn's Disease

Froehlich, F. (Lausanne/Basle); Juillerat, P.; Pittet, V.; Felley, C.; Mottet, C.; Vader, J.-P.; Michetti, P.; Gonvers, J.-J. (Lausanne)

136 Upper Gastrointestinal Crohn's Disease

Mottet, C.; Juillerat, P.; Pittet, V.; Gonvers, J.-J.; Michetti, P.; Vader, J.-P.; Felley, C. (Lausanne); Froehlich, F. (Lausanne/Basle)

141 Extraintestinal Manifestations of Crohn's Disease Juillerat, P.; Mottet, C.; Pittet, V. (Lausanne); Froehlich, F. (Lausanne/Basle); Felley, C.; Gonvers, J.-J.; Vader, J.-P.; Michetti, P. (Lausanne)

149 Pregnancy and Breastfeeding in Patients with Crohn's Disease Mottet, C.; Juillerat, P.; Pittet, V.; Gonvers, J.-J. (Lausanne); Froehlich, F. (Lausanne/Basle); Vader, J.-P.; Michetti, P.; Felley, C. (Lausanne)

161 Drug Safety in Crohn's Disease Therapy Juillerat, P.; Pittet, V.; Felley, C.; Mottet, C. (Lausanne); Froehlich, F. (Lausanne/Basle); Vader, J.-P.; Gonvers, J.-J.; Michetti, P. (Lausanne)

169 Author and Subject Index

\section{KARGER}

Fax +4161306 1234 E-Mail karger@karger.ch www.karger.com (c) 2008 S. Karger AG, Basel

Access to full text and tables of contents, including tentative ones for forthcoming issues: www.karger.com/dig_issues 


\section{No. 3-4}

Editorials

179 Human Smoking - Animal Passive Smoking: What Do We Learn from It about Inflammatory Bowel Disease? Reif, S. (Tel-Aviv)

221 Gastroesophageal Reflux and Atypical Symptoms: The Role of Impedance-pH Monitoring

Fornari, F.; Sifrim, D. (Leuven)

Reviews

171 Potential Options to Optimize Therapy of Gastroesophageal Reflux Disease with Proton Pump Inhibitors

Tonini, M. (Pavia); Vigneri, S. (Palermo); Neri, M. (Chieti); Cuomo, R. (Naples); Savarino, V. (Genoa); Pace, F. (Milan)

235 Wireless Capsule pH Monitoring: Does It Fulfil All Expectations? Maerten, P.; Ortner, M., Michetti, P.; Dorta, G. (Lausanne)

241 EndoCinch ${ }^{\circledR}$ Treatment for Gastro-Oesophageal Reflux Disease

Mahmood, Z. (Whitehaven); Ang, Y.S. (Manchester)

Original Papers

181 Effect of Passive Cigarette Smoking on Colonic $\alpha 7$-Nicotinic Acetylcholine Receptors in TNBS-Induced Colitis in Rats

Sun, Y.P.; Wang, H.H.; He, Q. (Beijing); Cho, C.H. (Hong Kong)

192 Manometric Evaluation of the Esophagus in Patients with Behçet's Disease

Bektas, M.; Altan, M.; Alkan, M.; Ormeci, N.; Soykan, I. (Ankara)

196 The c.1-260C $>$ T Promoter Variant of CD14 but Not the c.896A $>$ G (p.D299G) Variant of Toll-Like Receptor 4 (TLR4) Genes Is Associated with Inflammatory Bowel Disease Baumgart, D.C.; Büning, C.; Geerdts, L.; Schmidt, H.H.; Genschel, J.; Fiedler, T.; Gentz, E. (Berlin); Molnar, T.; Nagy, F.; Lonovics, J. (Szeged); Lochs, H.; Wiedenmann, B.; Nickel, R.; Witt, H.; Dignass, A. (Berlin)
203 A Multicenter Prospective Survey on Informed Consent for Gastrointestinal Endoscopy in China

Bai, Y.; Gao, J. (Shanghai); Yang, Y. (Yichang); Long, F. (Nanchong); Jin, H. (Zhoushan); Li, C. (Dongying); Zou, D.-W.; Li, Z.-S. (Shanghai)

207 Empirical Therapy for Symptomatic Gastroesophageal Reflux Disease in Primary Care: Determinants of Efficacy

Arts, J. (Leuven); Eisendrath, P.; Devière, J. (Brussels); Tack, J. (Leuven)

215 A New Eradication Resistance Index as a Predictor of Metronidazole-Containing Second-Line Treatment of Helicobacter pylori

Nishizawa, T.; Suzuki, H.; Masaoka, T.; Iwasaki, E.; Hibi, T. (Tokyo)

223 Combined pH-Metry/Impedance Monitoring Increases the Diagnostic Yield in Patients with Atypical Gastroesophageal Reflux Symptoms

Bajbouj, M.; Becker, V.; Neuber, M.; Schmid, R.M.; Meining, A. (Munich)

229 Perceptions and Beliefs concerning Gastroesophageal Reflux Disease: Physicians and Patients Disagree

Reimer, C.; Bytzer, P. (Køge)

248 Helicobacter pylori Activates Gastric Epithelial Cells to Produce Interleukin-8 via Protease-Activated Receptor 2 Kajikawa, H.; Yoshida, N.; Katada, K. (Kyoto); Hirayama, F. (Osaka); Handa, O.; Kokura, S.; Naito, Y.; Yoshikawa, T. (Kyoto)

256 Evaluation of Faecal Occult Blood Test and Lactoferrin Latex Agglutination Test in Screening Hospitalized Patients for Diagnosing Inflammatory and Non-Inflammatory Diarrhoea in Dhaka, Bangladesh

Ashraf, H. (Dhaka); Beltinger, J. (Basel); Alam, N.H.; Bardhan, P.K.; Faruque, A.S.G.; Akter, J.; Salam, M.A. (Dhaka); Gyr, N. (Basel)

Case Report

188 Percutaneous Thrombin Embolization of a Ruptured Peripancreatic Pseudoaneurysm

Ward, E.V.M.; Buckley, O.; Doody, O.; Govender, P.; Conlon, K.; Torreggiani, W.C. (Tallaght)

262 JGA News

263 Acknowledgement to Referees

265 Author Index Vol. 76, 2007

266 Subject Index Vol. 76, 2007 